\title{
Digital Mammography and Thermography Processing for Breast Cancer Detection
}

\author{
C.M. Gómez-Sarabia ${ }^{1}$, R. Guzmán Cabrera² ${ }^{2}$ J.R. Guzmán-Sepúlveda ${ }^{3}$, \\ M. Torres-Cisneros ${ }^{4}$, J. Ojeda Castañeda ${ }^{4}$ \\ ${ }^{1}$ Universidad de Guanajuato, Digital Arts Department, DICIS, Salamanca, Guanajuato, \\ Mexico \\ ${ }^{2}$ Universidad de Guanajuato, Electrical Engineering Department, DICIS, Salamanca, \\ Guanajuato, Mexico \\ ${ }^{3}$ University of Central Florida, CREOL, The College of Optics and Photonics, Orlando, FL, \\ USA \\ ${ }^{4}$ Universidad de Guanajuato, Electronics Department, DICIS, Salamanca, Guanajuato, \\ Mexico
}

maggie_gomezsa@yahoo.com,guzmanc81@gmail.com,jrafael_guzmans@yahoo.com.mx, mtorres37@me.com,jorge_ojedacastaneda@yahoo.com

\begin{abstract}
Primary prevention in early stages of breast cancer becomes crucial for diagnosis and, at the same time, complicated to achieve since the causes remain practically unknown. X-ray mammography is the most widely used screening technique due to cost effectiveness and its capability to provide valuable structural information thus allowing detection of characteristic cancer signatures such as masses and microcalcifications. Recently, thermography has gained a lot of interest since it has been demonstrated to be a non-invasive technique capable of revealing the health condition of the breasts in terms of physiological changes due to cancer formation. In this paper an approach based on intensity-based segmentation by means of morphological operators is proposed in order to detect regions potentially containing cancer in digital mammograms and thermograms. The algorithm is tested over several images taken from the Digital Database for Screening Mammography and the American College of Clinical Thermography, and the results suggest that the proposed algorithm is a suitable tool to successfully identify and extract regions of interest in a variety of environments and conditions, among which are several types of cancer and different image angles.
\end{abstract}

Keywords: breast cancer, digital image processing, mammography, thermography.

\section{Introduction}

Breast cancer is the most common cause of death in women and the second leading cause, after lung cancer, of death around the world [1-2]. The risk of a woman developing invasive breast cancer at some time in her life is about 12\% [3]: the National 
Cancer Institute of Canada estimates that 1 in 9 women will develop breast cancer and that 1 in 27 will die from this disease [4], while the incidence of developing breast cancer in the United Stated is estimated to be 1 in 8 women [5]. There is not an effective way to prevent and avoid breast cancer since the causes remain practically unknown; therefore, early detection plays a major role in diagnosis and treatment.

In this regard, the most recent advances in early detection include techniques such as digital mammography, tomosynthesis, contrast-enhanced mammography, screening ultrasound, and contrast-enhanced magnetic resonance imaging (MRI) [6]. None of these techniques have demonstrated to be completely satisfactory; however some specific conditions have been discovered to be determinant in the selection of the appropriate technique in order for the performance to be more effective.

For instance, MRI has been demonstrated to be more sensitive than mammography in high-risk patients and the combination of these two would be advisable in this case; screening ultrasound is suggested in patients having a high-risk condition who cannot tolerate MRI; and ultrasound screening together with supplemental screening techniques is recommended for intermediate-risk patients and women with dense breasts.

Some other imaging modalities for breast cancer screening, such as breast-specific gamma imaging and positron emission mammography, have not been supported mainly due to the radiation doses, which are 15 to 30 times higher than the dose from digital mammography [7].

In order to improve the accuracy and efficiency of the diagnosis in breast imaging, Computer-Aided Diagnosis (CAD) has been introduced within the screening process to support radiologists and internists in terms of image interpretation. CAD systems aim to complement the screening techniques and they can be found mainly in two forms depending on their purpose: Computer-Aided Detection (CADe) and Computer-Aided Diagnosis (CADx). The latter are oriented at image classification (i.e. malignancy, benignity) while CADe systems are focused on identifying regions of interest (i.e. suspicious regions) thus providing a second opinion on potentially abnormal regions that may contain breast cancer [8].

In this work, a CADe scheme is proposed to effectively analyze digital mammograms and thermograms based on texture segmentation for the detection of early stage tumors and cancerous activity. Regarding mammography, the algorithm was tested on several images taken from the Digital Database for Screening Mammography (DDSM) [9-10] while thermography images were taken from the website of the American College of Clinical Thermography (ACCT) [11].

The identification of the regions of interest (i.e. image segmentation) is done by means of morphological operators and the extraction is then performed through machine learning techniques and the clustering algorithm for intensity-based segmentation. In both cases the images were previously hand-labeled and classified by specialists thus allowing a reliable clinical baseline.

The results show that the proposed CADe algorithm is a suitable tool for qualitative evaluation of both digital mammograms and thermograms, since it allows effective processing to successfully extract the regions of interest where the characteristic signatures of breast cancer can be found. 


\section{Mammography and Thermography}

To date X-ray mammography is the most widely used technique in clinical practice due to its low cost and accessibility and it is actually the only method shown to decrease mortality in two-thirds of studies. Direct data shows a mortality decrease of $30 \%$ in the United States since the advent of mammography [12].

Although screening mammography exhibits some limitations, such as low reliability among young women or women who have undergone surgery, the debatable rate of "overdiagnosis", and the dependence of the sensitivity on the density of the breast tissue [13-16], it is recognized as the most effective method for early detection of breast cancer since it provides high sensitivity on fatty breasts and good performance on micro-calcification detection [17]. Some health, medical, and professional agencies and societies, among which are the American Cancer Society (ACS), the American College of Radiology (ACR), the Centers for Disease Control and Prevention (CDCP), the National Cancer Institute (NCI), and the Society of Breast Imaging (SBI) [18], agree with the Food and Drug Administration (FDA) [19] that mammography is the most effective method for breast cancer detection in its earliest, most treatable stages [20].

In addition to cost effectiveness and accessibility, mammography gained popularity in the last decade due to several improvements on image-processing methods proposed to address the problem of tumor detection. These improvements include a large variety of techniques among which the most widely studied are those based on wavelet transforms and machine learning. In terms of techniques based on wavelet transforms, proposals using fractal analysis, discrete wavelet transform, and Markov random fields can be found in the literature [21]. On the other hand, regarding machine learning techniques, some relevant proposals include a neural network architecture based on multiple circular path convolutions for the analysis of tumor and tumor-like structures [22], as well as a two-stage adaptive density-weighted contrast enhancement algorithm for tumor detection [23].

However, the great need for non-invasive methods makes screening techniques such as thermography of interest to specialists. Thermography is a biologically inert method that screens the temperature distribution across the skin surface and it is based on the biological characteristic of the temperature increase of the tissue due to a rise in the metabolic activity (i.e. carcinogenesis).

Studies on thermographic breast imaging have been reported prior [24] and recent studies demonstrate that thermography performs better compared to mammography: thermography has a sensitivity of $100 \%$, specificity of $79 \%$, and proportion of true results of $92 \%$, compared to sensitivity of $85 \%$, specificity of $84 \%$, and proportion of true results of $85 \%$ for mammography [6].

Unlike mammography, thermography has not been validated as a screening or adjunctive diagnostic tool, neither by the SBI nor the FDA, due to the lack of conclusive evidence and the sensitivity of the results to statistical treatment [25].

Nevertheless, the bottom line is that thermography is a non-invasive technique to monitor breast health (i.e. no radiation or breast compression involved) and it offers crucial advantages over mammography such as the capability to detect hormonal imbalances and physiologic changes related to cancer while in cellular phase, years before it is detectable by means of mammographic techniques. Furthermore, based on the fact that only $25 \%$ of the cases have a genetic component [12], the characteristics 
mentioned above make thermography a suitable assessment tool since the risk of developing breast cancer is significantly modifiable in early stages.

\section{Method}

The identification of regions of interest related to breast cancer is performed by means of texture analysis, which depends not only on the image itself but also on the purpose for which it is used [21]; and indeed, it has been demonstrated that the dataset itself can be significantly influential on the results [17].

The proposed algorithm deals with 8-bit gray-scale images obtained from the Digital Database for Screening Mammography (DDSM) [9-10] and the website of the American College of Clinical Thermography (ACCT) [11]. Based on the fact that abnormalities in the breast tissue are represented by regions of the image with their own properties, different from the general characteristics of the rest of the image [26-28], the image analysis is focused on regional properties such as texture and intensity variations. Texture differences, encoded in the transition regions, are then identified by means of morphological analysis and, in this particular case, an entropy-based algorithm is preferred over a gradient-based one due to the several intensity changes that take place in a single transition region [27].

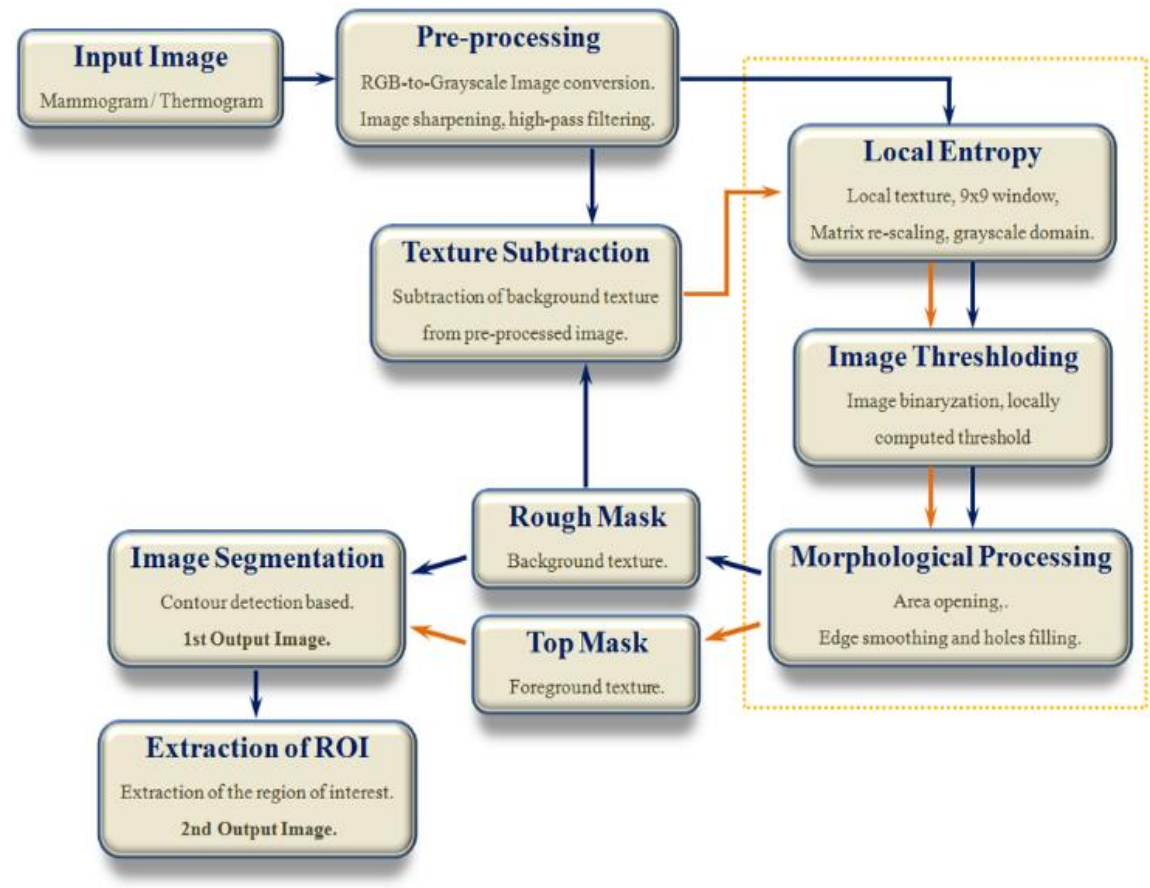

Fig. 1. General flowchart of the proposed algorithm.

Fig. 1 shows a general flowchart of the proposed algorithm containing the main stages of the process: image preprocessing, identification of the background texture, identification of the texture of the region of interest (ROI), and results display. 
The preprocessing stage consists of image sharpening, only, in order to enhance the details in the input image and it is not strictly required. The blocks inside the box are the most important ones since the texture analysis is performed in these stages; they will be further explained in detail. It can be noticed from the diagram that the background texture is used as a contrast mask to identify the ROIs, consequently the rough mask (i.e. background texture) directly determines what is identified as "abnormal" in the top mask (i.e. textures of ROIs).

The first critical stage in the proposed algorithm is the computation of the local entropy of the image. According to the literature, the local entropy of a small region $\boldsymbol{\Omega}_{\mathbf{k}}$ within the image, with dimensions (window size) $\mathbf{M}_{\mathbf{k}} \times \mathbf{N}_{\mathbf{k}}$, is defined as follows [27-28]:

$$
E\left(\Omega_{k}\right)=-\sum_{i=0}^{L-1} P_{i} \log \left(P_{i}\right)
$$

where:

$$
P_{i}=\frac{n_{i}}{M_{k} \times N_{k}}
$$

is the probability of occurrence of the discrete intensity $\mathbf{i}$ in the neighborhood $\boldsymbol{\Omega}_{\mathbf{k}}$ and $\mathbf{n}_{\mathbf{i}}$ is the number of pixels in the neighborhood that happen to have intensity of $\mathbf{i}$. $\mathbf{L}$ is the maximum discrete intensity and $\mathbf{E}\left(\boldsymbol{\Omega}_{\mathbf{k}}\right)$ is the local entropy of the windowed region.

The discrete intensity $\mathbf{i}$ is defined as a global parameter and can be adjusted to be close to the intensity of the region where the abnormality is suspected to be. A $9 \times 9$ sized window was used to compute the local entropy and the texture properties were defined only by the adjacent neighbors of the actual pixel.

After the local entropy of the image is computed using Eq. (1)-(2), a primitive version of the background texture is available, however, in order to use the background texture as a contrast mask, a binarization process takes place right after computing the local entropy.

Subsequent intermediate stages constituting the morphological treatment will determine the final version of the background texture. Firstly, a process of area opening is performed to remove the "small objects"; then edges smoothing (i.e. dilation followed by erosion) using a square 9x9 mask takes place; finally, the binary mask is filled by connecting the isolated background pixels.

The binary mask obtained in previous stages based on the background texture is now used to subtract the background component from the input image. After subtraction, the same texture analysis (stages inside the box) is performed over the non-background image using the same parameters than in the case of the background texture. The regions filtered by the binary mask (background) do not contribute to or affect the results related to the ROIs since the local entropy is minimal for regions consisting of pixels with the same intensity [29].

The morphological treatment of the binary image obtained for the ROIs consists of the same intermediate processes than in the previous case but, unlike the background texture case, the thresholding of the non-background region is performed using a local threshold considering the image where the background is ignored. Once the binary 
mask of the ROIs is obtained, the contours of the ROIs are outlined for the output (i.e. segmented and extracted), and images are displayed.

\section{$4 \quad$ Results}

The proposed algorithm was tested on both mammographic and thermographic images that allow qualitatively illustration of its performance under a variety of conditions.

As mentioned before, the input images in all cases are 8-bit gray-scale images; in the case of mammograms, the images were obtained from the Digital Database for Screening Mammography (DDSM) [9-10] while the thermograms were obtained from the website of the American College of Clinical Thermography (ACCT) [11].

\subsection{Breast Mammography}

The mammograms upon which the proposed algorithm was tested were previously analyzed, diagnosed, classified, and hand-labeled by specialists. The images processed include marks indicating the actual location of the lesions but, as it will be seen later, the algorithm is insensitive to the hand-made label and the ROIs can be successfully identified and extracted.

Fig. 2 shows the main results of the segmentation and extraction processes for a mammogram diagnosed with malign cancer. Fig. 2a shows the original image, which highlights the abnormal region, while Figs. 2b-c show the segmented and extracted images. The original image was converted from RGB to gray-scale by forming a weighted sum of the $\mathrm{R}, \mathrm{B}$, and $\mathrm{G}$ components with the respective standard coefficients $0.2889,0.5870$, and 0.1440 .
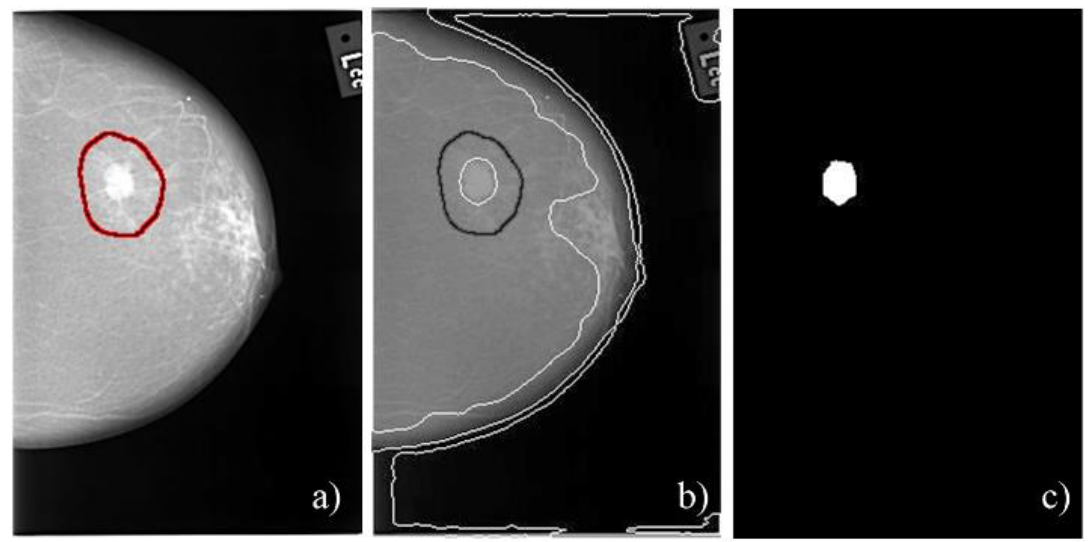

Fig. 2. Digital image processing of a mammogram diagnosed with malign breast cancer. (a) Original hand-labeled image, (b) segmented image, and (c) extracted image. Volume 02 Case C0037 - Left CC [9-10].

In this particular case, the segmentation and extraction were found to be successful for a relative threshold intensity of 0.750 and a reference opened area of 250 pixels. 
One single ROI, with area of 535 pixels, was successfully extracted and, as can be confirmed by Fig. 2b, the hand-made label does not contribute, interfere, or affect the segmentation: the contour of the ROI can be outlined independently from the label.
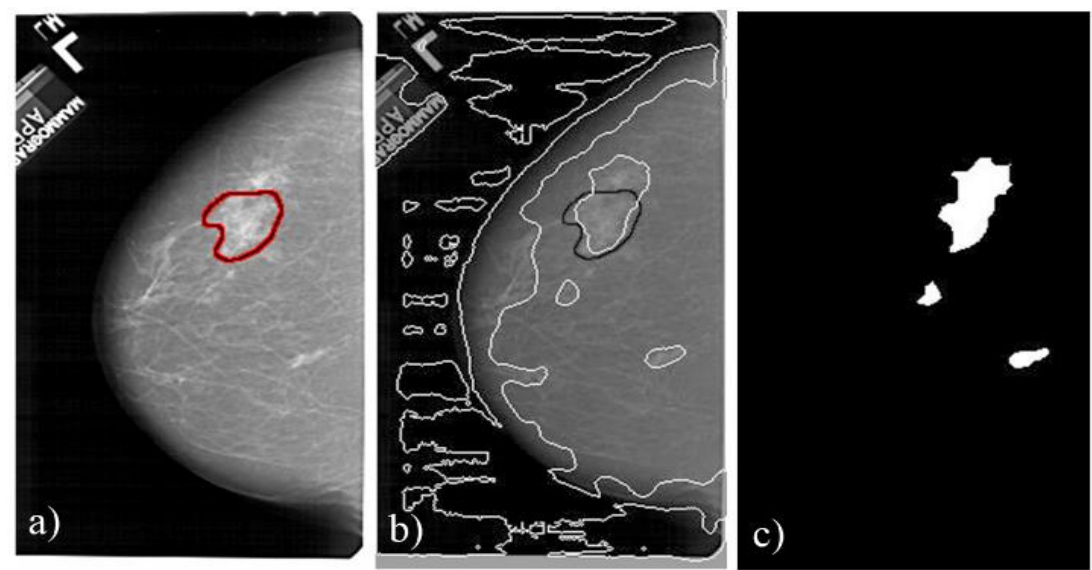

Fig. 3. Digital image processing of a mammogram diagnosed with benign breast cancer. (a) Original hand-labeled image, (b) segmented image, and (c) extracted image. Volume 02 Case A1237 - Left CC [9-10].

The fact that the ROI found by the proposed algorithm is smaller than that defined by the hand-made label can be explained based on the fact that the label encloses a larger area than that occupied by the lesion while the lesion in this case is well defined, significantly non-outspread, and located over a region with considerably clear boundaries, appreciable by naked eye.

The second case study is a mammogram diagnosed with benign cancer. Fig. 3 shows the results of the segmentation and extraction processes. In similar fashion to the previous case, Fig. 3a shows the original image, which highlights the lesion, while Figs. $3 \mathrm{~b}-\mathrm{c}$ show the segmented and extracted images, respectively. The original image was converted from RGB to gray-scale using the same standard set of coefficients.

Contrary to the previous case, the lesion is not clearly defined and the hand-made label encloses an outspread region with undefined boundaries. However, the segmentation and extraction processes were found to be successful for a relative threshold intensity of 0.762 and a reference opened area of 200 pixels.

In this case, three different ROIs were found over the image with similar texture properties. The total area of the ROIs is 1763 pixels and, once again, the ROIs can be successfully identified independently from the hand-made label.

Unlike the previous case, the largest ROI found extends over a considerably larger region than that defined by the hand-made label. Moreover, the proposed algorithm found two more regions having the same texture than that from the lesion that were not previously identified. This does not mean that the additional regions also contain benign cancer but it means that in terms of texture characteristics these regions have similar properties; further analysis is required to diagnose the additional regions. 


\subsection{Breast Thermography}

The thermograms over which the proposed algorithm was tested were previously analyzed, diagnosed, and classified by specialists; in this case the images are not handlabeled.

The fact that the thermogram screens the temperature distribution across the skin surface and not the structural properties of the breast tissue leads to specific implications that are to be taken into account further in the processing. The value of the threshold intensity around which the texture analysis is performed is expected to be higher only due to the color scale used in the pseudo-coloring process: the higher temperatures, meaning the regions containing the lesions, are closer to white.
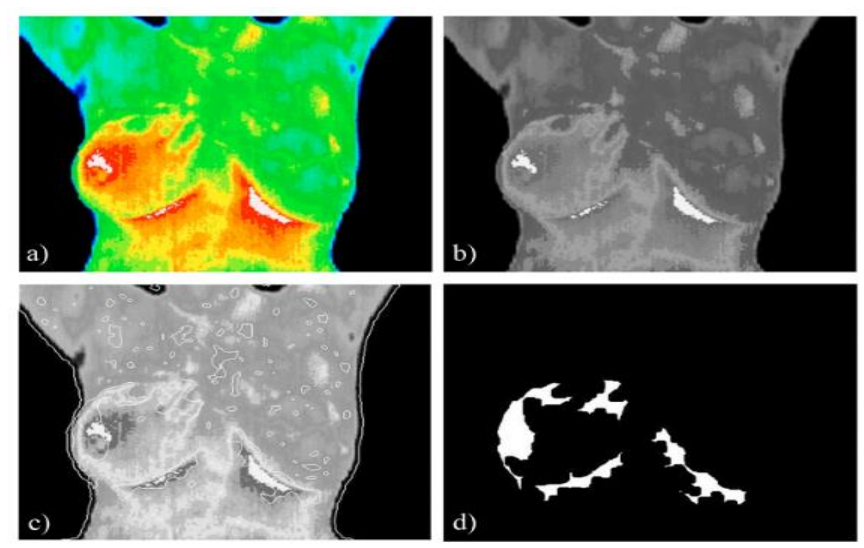

Fig. 4. Digital image processing of a thermogram diagnosed with inflammatory breast cancer [11]. a) Original pseudo-colored image, b) gray-scale image, c) segmented image, and d) extracted image.
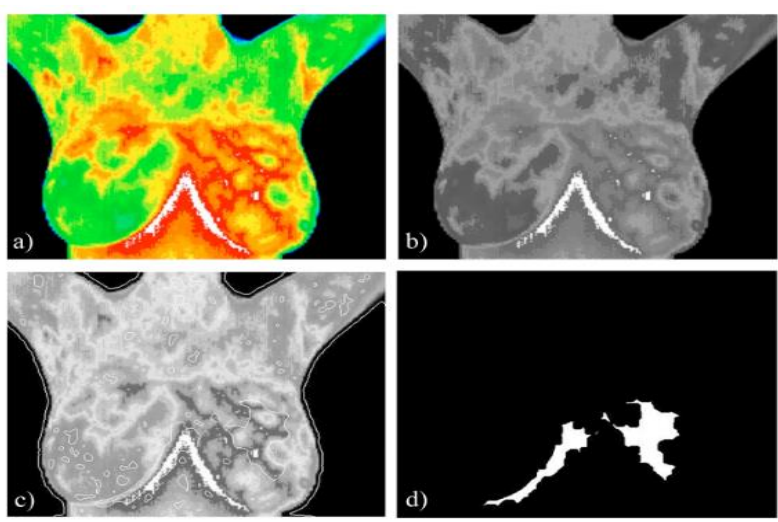

Fig. 5. Digital image processing of a thermogram diagnosed with breast fibrosis [11]. a) Original pseudo-colored image, b) gray-scale image, c) segmented image, and d) extracted image.

On the other hand, in terms of the ROIs themselves, much larger regions are expected to be extracted due to the fact that temperature distributions gradually extend 
over larger areas, leading to smooth transition regions between the background and the ROIs, then resulting in outspread and geometrically undefined regions.

Fig. 4 shows the main results of the segmentation and extraction processes for a thermogram diagnosed with inflammatory breast cancer. Fig. 4a-b show the original pseudo-colored and gray-scale images, respectively; while Figs. 4b-c show the processed images, segmented and extracted, respectively. The original pseudo-colored image was converted from RGB to gray-scale using the standard set of coefficients mentioned before.

In this particular case, the segmentation and extraction were found to be successful for a relative threshold intensity of 0.830 and a reference opened area of 1000 pixels. Several ROIs, with total area of 8060 pixels, were successfully extracted.

The second case study is a thermogram diagnosed with breast fibrosis. Fig. 5 shows the main results of the segmentation and extraction processes. Fig. 5a-b show the original pseudo-colored and gray-scale images, respectively, while Figs. 5b-c show the segmented and extracted processed images, respectively. The original pseudo-colored image was converted from RGB to gray-scale using the same standard set of coefficients.

In this particular case, the segmentation and extraction were found to be successful for a relative threshold intensity of 0.840 and a reference opened area of 1400 pixels. Several ROIs, with total area of 6885 pixels, were successfully extracted.

\section{Conclusions}

In this paper a texture segmentation approach is demonstrated for the detection of regions potentially containing cancer in both digital mammograms and thermograms. The proposed Computer-Aided Detection (CADe) system, consisting mainly of intensity-based segmentation by means of morphological operators, was tested on several images from the Digital Database for Screening Mammography and the American College of Clinical Thermography. Both cases have a reliable clinical baseline as the images were previously hand-labeled and classified by specialists.

In the case of mammography two case studies, diagnosed with malign and benign cancer, respectively, are reported; while regarding thermography, the case studies reported are diagnosed with inflammatory cancer and fibrosis, respectively. This variety of cases allows testing the effectiveness and robustness of the proposed algorithm; the obtained results show actual, successful, segmentation and extraction of the regions of interest in all the cases.

The input parameters of the algorithm are reference values of the normalized intensity and area of the regions that are to be found. In this regard, there are significant differences for mammograms and thermograms, due only to the nature of the screening techniques: both the reference intensity and the area are significantly larger in the case of thermograms due to the pseudo-coloring and the smoothness of the transitions between the background and the ROIs, respectively.

In the particular case of mammography, the mammogram diagnosed with malign cancer was successfully processed and the extracted ROI just confirmed the handlabeling. Unlike this case, the mammogram diagnosed with benign cancer does not have a well-defined lesion and, in fact, the extracted ROI extends over a larger area than that 
of the hand-labeling. Moreover, as a result of the processing, additional ROIs not present in the hand-labeling were found to have identical texture properties than those of the lesion.

In the case of thermography, both case studies present thermograms with large ROIs having smooth transition regions between ROIs and background. The algorithm still operates successfully over the thermograms and, despite that the ROIs do not have well defined boundaries, a significant correlation between the original and extracted images can be qualitatively depicted.

Overall, the proposed CADe algorithm is demonstrated to be stable and robust while processing breast images with a variety of different characteristics resulting in a reliable and suitable tool for the qualitative evaluation of both digital mammograms and thermograms.

\section{References}

1. Deserno, T. M.: Biomedical Image Processing. Springer, Berlin (2010)

2. Garcia, M., Jemal, A., Ward, E., Center, M., Hao, Y., Siegel, R., Thun, M.: Global, Cancer Facts \& Figures. American Cancer Society, Atlanta, GA (2007)

3. Breast Cancer Statistics. Available: http://www.breastcancer.org (2009)

4. Canadian cancer statistics. Canadian Cancer Society/National Cancer Institute of Canada, available: http://www.cancer.ca or http://www.ncic.cancer.ca (2013)

5. Probability of breast cancer in American women. National Cancer Institute, available: http://www.cancer.gov/cancertopics/factsheet/Detection/probability-breast-cancer (2013)

6. Kolarić, D., Herceg, Z., Nola, I. A., Ramljak, V., Kuliš, T., Katančić-Holjevac, J., Antonini, S.: Collegium Antropologicum. 37(2), pp. 583-588 (2013)

7. Mainiero, M. B., Lourenco, A., Mahoney, M. C., Newell, M. S., Bailey, L., Barke, L. D., Haffty, B. G.: J. Am. Coll. Rad. 10(1), pp. 11-14 (2013)

8. Giger, M. L., Karssemeijer, N., Schnabel, J. A.: Annual review of biomedical engineering. 0 (2013)

9. Heath, M., Bowyer, K., Kopans, D., Moore, R., Kegelmeyer, W. P.: The Digital Database for Screening Mammography. In: Proceedings of the Fifth International Workshop on Digital Mammography, M.J. Yaffe, ed., Medical Physics Publishing, pp. 212-218 (2001)

10. Heath, M., Bowyer, K., Kopans, D., Kegelmeyer, W. P., Moore, R., Chang, K., MunishKumaran, S.: Current status of the Digital Database for Screening Mammography. In: Proceedings of the Fourth International Workshop on Digital Mammography, pp. 457-460 (1998)

11. http://www.thermologyonline.org/index.html, American College of Clinical Thermography (ACCT).

12. Hudson, T., Poulsen, C. A.: Brush and Quill Productions. Journey to Hope, 2nd Ed (2011)

13. Calonge, N., Petitti, D. B., DeWitt, T. G., Dietrich, A. J., Gregory, K. D., Grossman, D., Wilt, T.: Annals of Internal Medicine. 151(10), pp. 716-W236 (2009)

14. Kopans, D. B., Smith, R. A., Duffy, S. W.: Radiology. 260(3), pp. 616-620 (2011)

15. Smith, R. A., Duffy, S. W., Tabár, L.: Oncology. 26(5), pp. 471-475 (2012)

16. Jørgensen, K. J., Keen, J. D., Gøtzsche, P. C.: Radiology. 260(3) pp. 621-627 (2011)

17. Bottema, M. J., Lee, G. N., Lu, S.: Series in Machine Perception and Artificial Intelligence. 39, pp. 17-54 (2000)

18. Food and Drug Administration. FDA, available: http://www.fda.gov

19. Society of Breast Imaging. SBI, available: http://www.sbi-online.org 
20. http://www.fda.gov/MedicalDevices/Safety/AlertsandNotices/ucm257259.htm

21. Zheng, L., Chan, A. K.: IEEE Trans. Med. Imaging. 20(7), pp. 559-567 (2001)

22. Li, H., Shih-Chung, B.L., Wang, Y., Kinnand, L., Freedman, M. T.: IEEE Trans. Med. Imaging. 21(2), pp. 150-158 (2002)

23. Chan, H. P., Petrick, N., Sahiner, B.: Computer-aided breast cancer diagnosis: artificial intelligence techniques in breast cancer diagnosis and prognosis series in machine perception and artificial intelligence. World Scientific Publishing Co. Pte. Ltd., 39, pp. 179-264 (2000)

24. Feig, S. A., Shaber, G. S., Schwartz, G. F., Patchefsky, A., Libshitz, H. I., Edeiken, J., Wallace, J. D.: Radiology. 122(1), pp. 123-127 (1977)

25. Brkljačić, B., Miletić, D., Sardanelli, F.: Collegium Antropologicum. 37(2), pp. 589-593 (2013)

26. Gerbrands, J. J.: Segmentation of noisy images. Ph.D. Dissertation, Delft University, The Netherlands (1988)

27. Yan, C., Sang, N., Zhang, T.: Pattern Recognition Lett. 24, pp. 2935-2941 (2003)

28. Gonzalez, R. C., Woods, R. E., Eddins, S. L.: Digital Image Processing Using MATLAB. Prentice Hall, Chap. 11 (2003)

29. Thum, C.: Optica Acta: Int. J. Optics, 31(2), pp. 203-211 (1984) 